\title{
Thermogravimetric investigation of sol-gel microspheres doped with aqueous glycerol
}

\author{
Rosaria Ciriminna, Giuseppe Pantaleo, Rosa La Mattina and Mario Pagliaro*
}

\begin{abstract}
Background: Silica-based microspheres encapsulating aqueous glycerol are promising curing agents affording the formation of better one-component polyurethane foams, namely thermoset polymers cured by atmospheric moisture that are widely and increasingly utilized in the construction industry. The use of renewable, non toxic glycerol from biodiesel and oleochemicals industry to cure PU foams in place of traditionally employed oil-derived mono and diethylene glycols is both technically and environmentally beneficial. The higher amount of hydroxyl groups in glycerol compared to both mono- and diethylene glycol results in considerably lower percentage of free monomeric methylene diphenyl diisocyanate (MDI) and higher crosslinking density of the cured foam.

Results: We investigate by thermogravimetric analysis the nature and amount of the microencapsulated species in sol-gel silica and organosilica microspheres encapsulating aqueous glycerol. Along with a percentage of glycerol in weight up to 35\%, the microspheres contain about $5 \mathrm{wt} \%$ water and $4 \mathrm{wt} \%$ entrapped surfactant.

Conclusions: The investigation shows the efficacy of the surfactant-assisted sol-gel microencapsulation aqueous glycerol in silica as well as in methyl-silica particles at least until 10\% degree of methylation. The results are relevant to the practical development of functional materials that can be used to cure better and greener polyurethane foams largely employed as coatings, adhesives and sealants in many industrial sectors.
\end{abstract}

Keywords: PU foams, Curing, Sol-gel microencapsulation, One-component foam, Solid curing agents

\section{Background}

We have recently described in this Journal the promising results of detailed testing of the GreenCaps in the formation of greener one component polyurethane foams (OCFs) under standard conditions [1]. Proposed as potential new solid curing agents, the GreenCaps are comprised of microporous silica-based microspheres encapsulating aqueous glycerol [2]. Adding to their environmental merit, the GreenCaps microparticles, in addition, are made using biodegradable, non toxic surfactant sorbitan oleate (Span 80) derived from natural products oleic acid and sorbitol [3].

Self-expanding OCFs cured by atmospheric moisture [4] are increasingly used in the construction industry, as sealants as well as adhesives to fix polystyrene and polyurethane panels to walls and insulate buildings. OCFs are commercially available in pressurized cans at retail

\footnotetext{
*Correspondence: mario.pagliaro@cnr.it

Istituto per lo Studio dei Materiali Nanostrutturati, CNR, via U. La Malfa 153, 90146 Palermo, PA, Italy
}

locations. Global production in 2014 rate exceeded 500 million units, growing at $4 \%$ yearly rate [5].

In general, the pressurized can comprises methylene diphenyl diisocyanate (MDI) along with a polyol blend added in the required amount for optimal "curing" up to the state in which no free isocyanate $(-\mathrm{NCO})$ groups are present in the thermoset final polymer. MDI is toxic and after application uncured isocyanates can cause eye and lung irritation, and chemical sensitization when absorbed through the skin [6]. It is therefore an important safety issue of the global PU industry that all isocyanate groups be fully reacted or "cured" so as to prevent health and safety hazard [7].

The use of glycerol as component of the polyol blend (the Component A at OCF producers) to cure PU foams in place of oil-derived polyols is rapidly increasing $[8,9]$, as its historically high price dropped following large overproduction as by-product from the biodiesel and oleochemicals manufacturing [10]. The higher amount of hydroxyl groups in the glycerol molecule (compared 
to monomeric glycols) results in considerably lower percentage of free monomeric MDI and higher crosslinking of the cured foam, which in its turn results in less curing shrinkage and less outflow of the sprayed material. Perhaps not surprisingly, PU foams made from $100 \%$ crude glycerol-based polyols biodegrade at faster rate than PU foams obtained from petroleum-based polyols [11].

We now report a detailed TGA/DSC analysis of these materials whose outcomes point to a number of findings of relevant practical value in the route towards commercialization of greener PU cans using the GreenCaps as solid curing agents.

\section{Experimental Reagents}

Glycerol, tetraethylorthosilicate (TEOS), methyltriethoxysilane (MTEOS), $n$-hexane, decalin (decahydronaphthalene mixture of cis + trans isomers), $37 \mathrm{wt} \%$ hydrochloric acid $(\mathrm{HCl})$ and sorbitane monooleate (Span 80$)$ were purchased by Sigma Aldrich. All chemicals were used as received, without further purification.

\section{Materials synthesis}

The micron-sized silica and organosilica microspheres were synthesized via sol-gel hydrolytic polycondensation of TEOS and MTEOS carried out in the droplets of a water-in-oil $(\mathrm{W} / \mathrm{O})$ microemulsion containing emulsified aqueous glycerol. Solvent $n$-hexane or decalin were employed as continuous organic phase, and non ionic Span 80 (sorbitan monooleate) as surfactant.

We have already described elsewhere [1] the preparation of the $\mathrm{SiO}_{2}$ spheres (GC7), and of the 5\% methylmodified silica microparticles (CG8). In the following we describe the synthesis of the $10 \%$ methylated microspheres (CG14). Furthermore, a blank material (CGB) made of $\mathrm{SiO}_{2}$ spheres without glycerol was prepared following the procedure used for sample CG7. The main parameters of the studied materials are summarized in Table 1.

In order to maximize the silane hydrolysis and subsequent condensation of hydrolysed silanes, for all samples the molar ratio $r=\mathrm{H}_{2} \mathrm{O}: \mathrm{Si}$ between water and the hydrolysable -OEt groups was set at $r=12$.

Table 1 Degree of alkylation and initial amount of glycerol used in the preparation of the silica and methylsilica microspheres investigated ${ }^{\mathrm{a}}$

\begin{tabular}{lcc}
\hline Sample & Degree of methylation $/ \mathbf{m o l} \%$ & Glycerol $\mathbf{g}$ \\
\hline CGB & 0 & 0 \\
CG7 & 0 & 15 \\
CG8 & 5 & 15 \\
CG14 & 10 & 5 \\
\hline
\end{tabular}

${ }^{\mathrm{a}}$ From a W/O microemulsion comprising TEOS or TEOS + MTEOS precursors with Span80 as surfactant and decalin as oil phase.
In detail, the CG14 sample was synthesized starting from a W/O microemulsion comprising glycerol (5 g) dissolved in water $(30 \mathrm{~mL})$, dispersed in a mixture of Span 80 in decalin previously obtained by fast stirring the surfactant in the oil phase at 9,500 rpm with an Ultra-Turrax T-25 with S 25 KR-18G dispersing tool (IKA).

A mixture of MTEOS (10\% in molar terms) and TEOS (90\% in molar terms) previously hydrolysed at $\mathrm{pH}=3$ (with $\mathrm{HCl} 0.05 \mathrm{~N}$ ) was added dropwise to the aqueous glycerol emulsion under constant stirring with an overhead rotor at $300 \mathrm{rpm}$. After addition of the alkoxides was complete, the mixture was stirred at $25^{\circ} \mathrm{C}$ for $1 \mathrm{~h}$ rising the overhead rotor speed at $530 \mathrm{rpm}$. The temperature was then increased stepwise, first to $75^{\circ} \mathrm{C}$ and then to $100^{\circ} \mathrm{C}$ keeping the mixture each time at the newly set temperature for $1 \mathrm{~h}$. The precipitate thereby obtained was filtered through a glass filter, washed extensively with decalin and mildly dried in an oven at $50^{\circ} \mathrm{C}$. A pale yellow powder was eventually isolated.

\section{Materials analysis}

The thermogravimetric analysis was performed on a Mettler Toledo TGA/DSC1, under nitrogen using the following heating profile: step $1\left(25^{\circ} \mathrm{C}-100^{\circ} \mathrm{C}\right.$ at $\left.10^{\circ} \mathrm{C} / \mathrm{min}\right)$; step $2\left(100^{\circ} \mathrm{C}\right.$ for $\left.30 \mathrm{~min}\right)$; step $3\left(100^{\circ} \mathrm{C}-400^{\circ} \mathrm{C}\right.$ at $5^{\circ} \mathrm{C} /$ $\mathrm{min})$; step $\left(400^{\circ} \mathrm{C}-1100^{\circ} \mathrm{C}\right.$ at $\left.10^{\circ} \mathrm{C} / \mathrm{min}\right)$. The plateau at $100^{\circ} \mathrm{C}$ was applied to ensure complete removal of water.

\section{Results and discussion}

Figure 1 shows that the 10\%-methyl modified CG14 microspheres obtained from prehydrolysed TEOS + MTEOS mixtures are spherical and well isolated, with a size around $60 \mu \mathrm{m}$ (Figure 1). We remind here that the low HLB

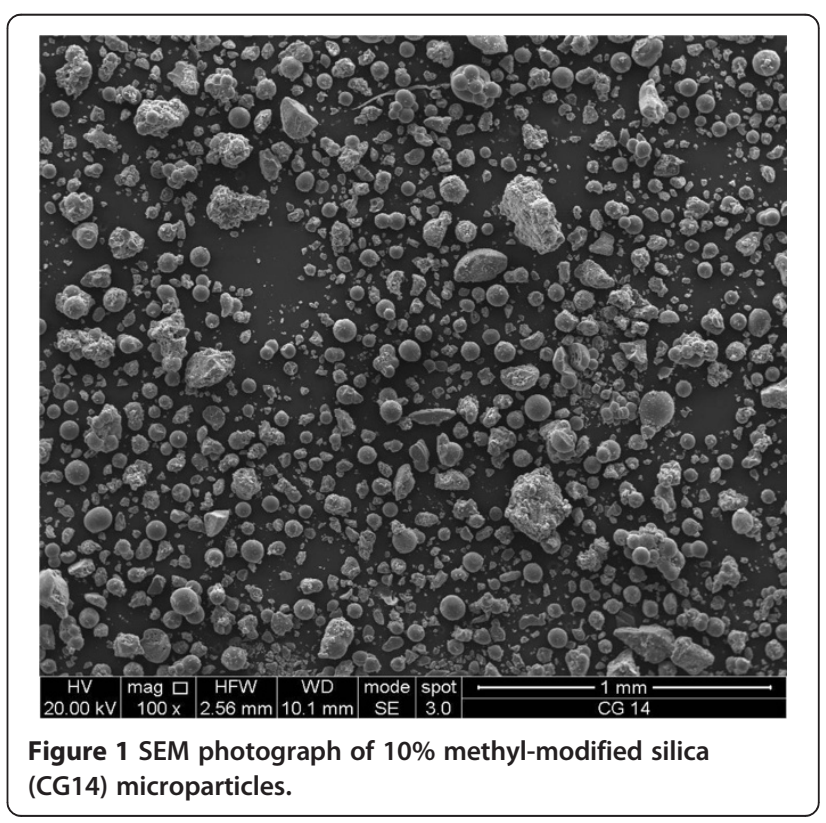




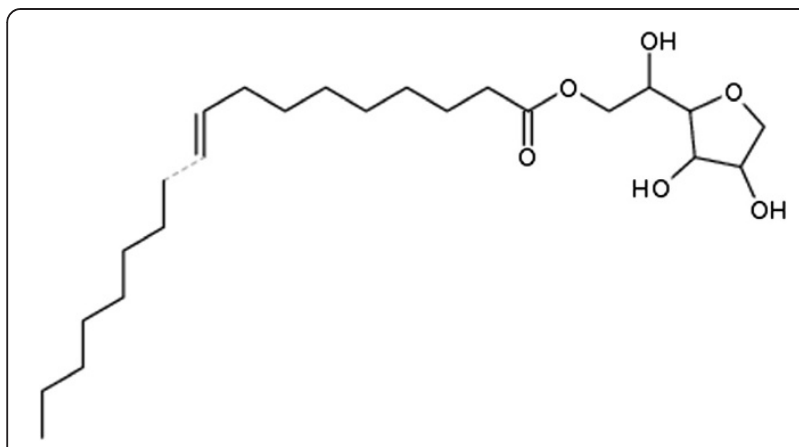

Figure 2 Chemical structures of Sorbitan oleate (Span 80).

(hydrophilic-lipophilic balance) value of hydrophobic surfactant sorbitan oleate (Figure 2, HLB = 4.3) [12] facilitates the formation of the hydrophilic droplets and the production of spherical particles [13].

The SEM photograph of CG14 material in Figure 1 shows that non-spherical aggregates are formed upon the mild drying step of the sol-gel precipitate. Besides a few broken microspheres, the larger particles are comprised of aggregated microspheres. Such aggregation is avoided in industrial applications of sol-gel microparticles by leaving the as-formed microspheres suspended in aqueous or in organic solvent. This is the case, for instance, of the Eusolex- $U V$ microcapsules that are delivered by Merck to customer cosmetic companies as a suspension of silica microparticles in water [14].

The nature and amount of the microencapsulated species was assessed from dynamic thermogravimetric analysis (TGA) and differential thermal analysis (DTA). Experiments were carried out under $\mathrm{N}_{2}$ atmosphere using both doped and blank microparticles, as well as pure glycerol, decalin and Span 80.

Figure 3 shows the TGA/DSC profiles of $\mathrm{SiO}_{2}$ microspheres (blank, CGB).

Figures 4, 5 and 6 display, respectively, the TGA/DSC profile of $\mathrm{SiO}_{2}$ (CG7), 5\%-methyl modified silica (CG8) and 10\%-methyl modified silica (CG14) microparticles doped with aqueous glycerol.

Finally, the TGA/DSC profiles of pure glycerol, decalin and surfactant Span 80 are displayed in Figures 7, 8 and 9.

In the blank sample CGB (Figure 3), the first weight loss due to water evaporation $(-8.5 \%)$ is followed by a similar weight loss peak for decalin at $185^{\circ} \mathrm{C}$. Eventually, at $507^{\circ} \mathrm{C}$ also the entrapped surfactant molecules are released $(-3.8 \%)$ and thermally decompose.

In the case of samples encapsulating glycerol, again the first weight loss is due to water evaporation followed by a midpoint around $210^{\circ} \mathrm{C}$. Said weight losses occurring at $\mathrm{T}<250^{\circ} \mathrm{C}$ correspond to endothermic processes. The endothermic peak centered at around $200^{\circ} \mathrm{C}$ (see lower panel in Figures 4, 5 and 6) is similar in shape and intensity for all glycerol-containing samples, confirming that the corresponding weight loss is in each case associated to evolving glycerol.

Comparison with the TGA profile of pure glycerol (Figure 7) shows that microencapsulated glycerol evolves at $20^{\circ} \mathrm{C}$ lower temperature, which is in agreement with the dispersion of tightly hydrogen-bonded glycerol molecules adsorbed at the silica and organosilica surface.

A consistent efficacy in the encapsulation of the hydrophilic glycerol molecules within the inner porosity in either $\mathrm{SiO}_{2}$ and in ORMOSIL (organically modified silica)

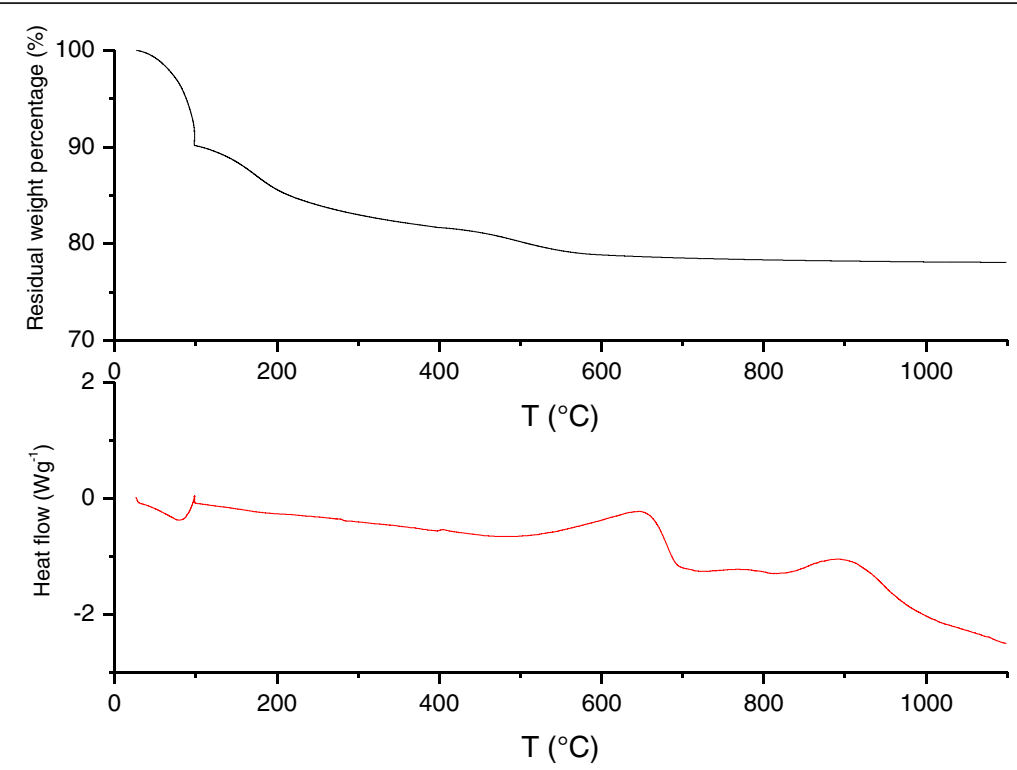

Figure 3 TGA/DSC of CGB microparticles. 


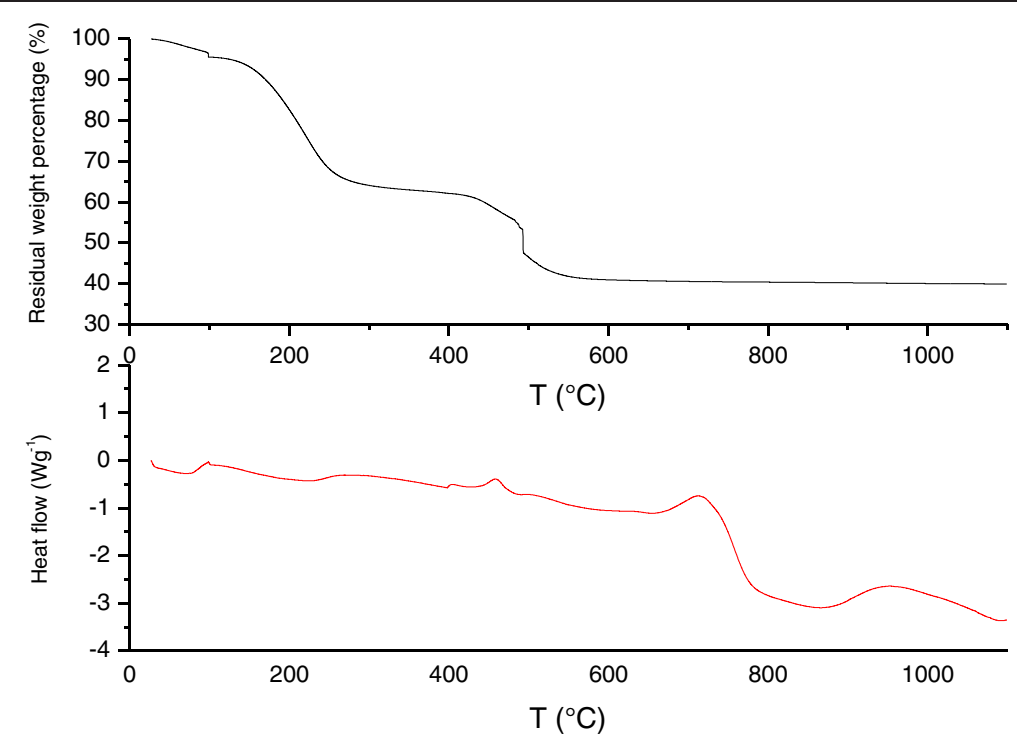

Figure 4 TGA/DSC of CG7 microparticles.

microparticles is observed. The amount of entrapped glycerol varies between $33 \mathrm{wt} \%$ for $\mathrm{SiO}_{2}$ (CG7) and $35 \mathrm{wt} \%$ for 5\%-methylsilica (CG8) microparticles. This outcome confirms that glycerol is encapsulated within the large inner porosity and not adsorbed at the external surface (in the latter case, indeed, the amount of liberated glycerol would be below $3-5 \mathrm{wt} \%$ ).

The TGA profile of the CG14 microspheres (Figure 6) shows that now $11 \mathrm{wt} \%$ of the resulting microspheres is comprised of glycerol. For this material, however, only $5 \mathrm{~g}$ of glycerol were used in the microencapsulation process in place of $15 \mathrm{~g}$ of glycerol employed in the preparation of the
CG7 and CG8 samples. This outcome, even though two points only do not allow to derive a general correlation, seems to suggest a linear variation in the fraction of glycerol entrapped $v s$ glycerol in solution. We remind here that, even though the polarity of ORMOSIL cages diminishes linearly with the amount of organotrialkoxysilane precursor $\mathrm{MeSi}(\mathrm{OEt})_{3}$ co-condensed with TEOS [15], the porosity of methyl-modified ORMOSIL shows the most polar microenvironment compared to any other organotrialkoxysilanes $\mathrm{R}-\mathrm{Si}(\mathrm{OEt})_{3}$ co-condensed with TEOS [16].

The TGA profiles of all microencapsulated glycerol samples show that all microspheres encapsulate sorbitan

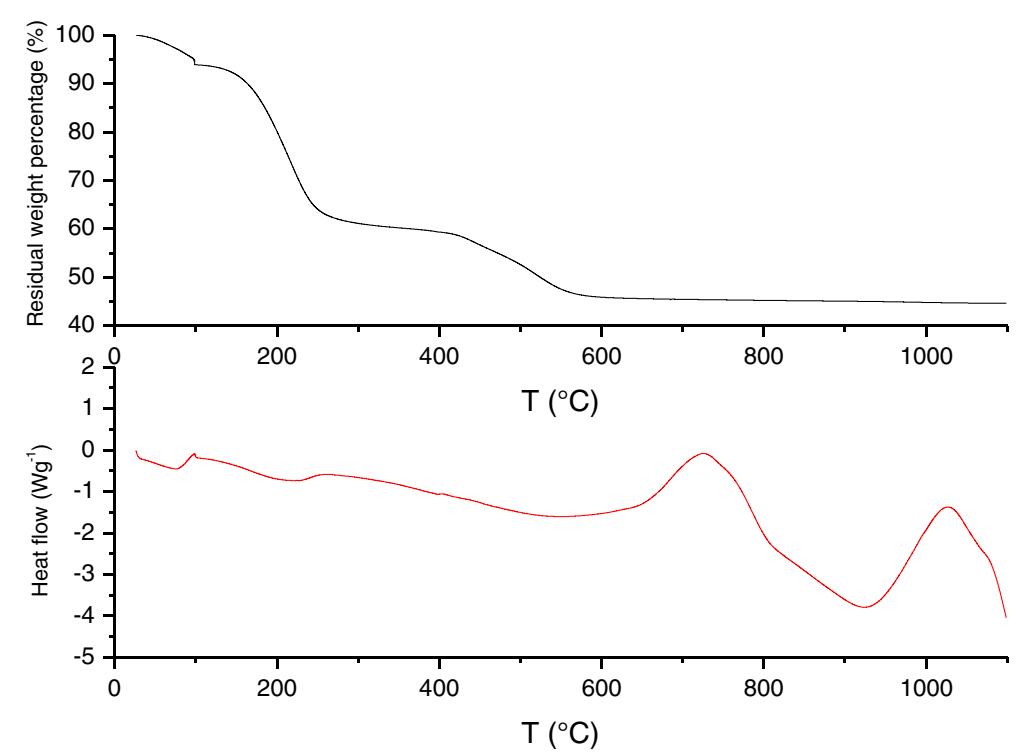

Figure 5 TGA/DSC of CG8 microparticles. 


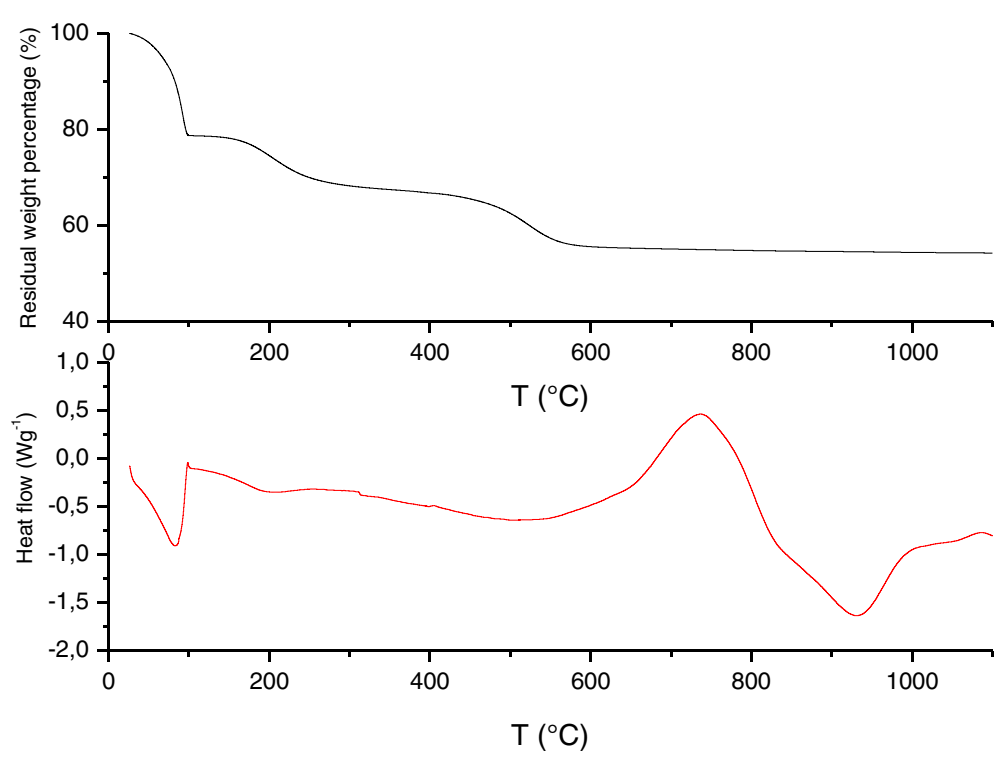

Figure 6 TGA/DSC of CG14 microparticles.

monoleate within their inner porosity with almost constant $4 \%$ in weight of the microparticles being made of Span 80 . The latter surfactant is highly soluble in decalin. Yet, prolonged washing of the asobtained microparticles with decalin is not capable to eliminate all the surfactant, showing that the residual surfactant is tightly entrapped within the inner porosity of the silica-based microspheres.

Finally, all samples show 40-45 wt\% residual inorganic content made of non porous $\mathrm{SiO}_{2}$ formed upon condensation of virtually all $\mathrm{Si}-\mathrm{OH}$ at the surface (with water elimination and consequent loss of porosity).
A similar behavior - namely a first significant weight loss due to water evaporation was followed by another significant weight loss peak for glycerol - was observed by Galgali and co-workers in the thermogravimetric analysis of coreshell microcapsules encapsulating aqueous glycerol in a thin layer of $\mathrm{SiO}_{2}$ [17]. In the latter case, the core content of the microcapsules was $25 \mathrm{wt} \%$ of water, $50 \mathrm{wt} \%$ glycerol and $25 \mathrm{wt} \%$ residual inorganic content.

In the present case of full silica and organosilica microspheres, the residual inorganic content of silica is around $40 \mathrm{wt} \%$, almost twice higher compared to core/shell microcapsules.

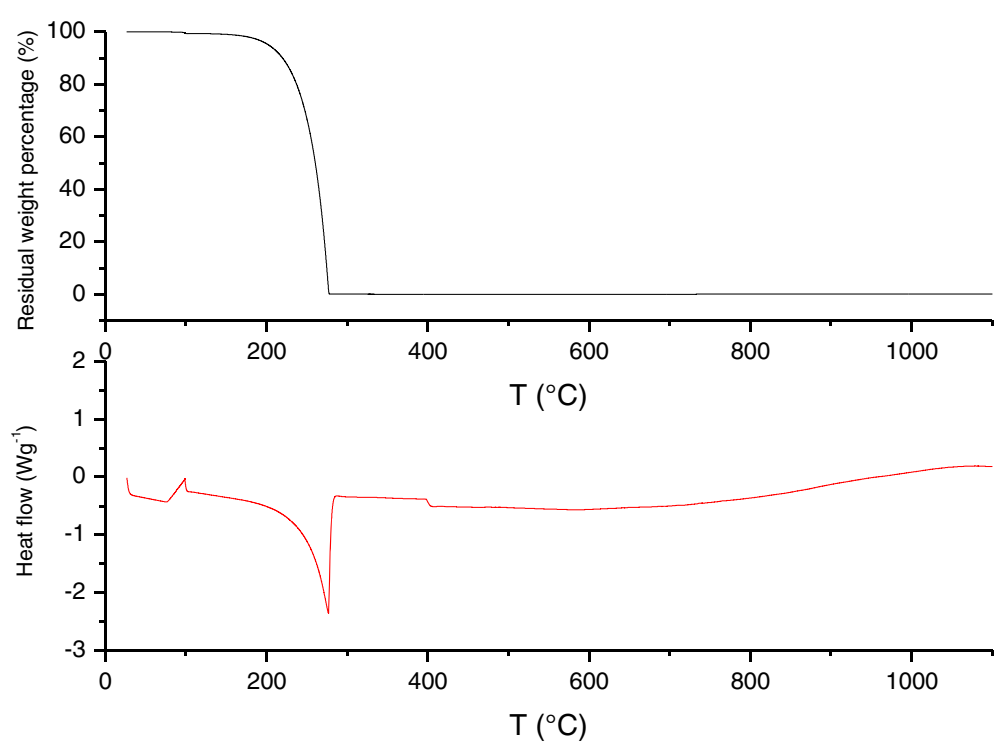

Figure 7 TGA/DSC of glycerol. 


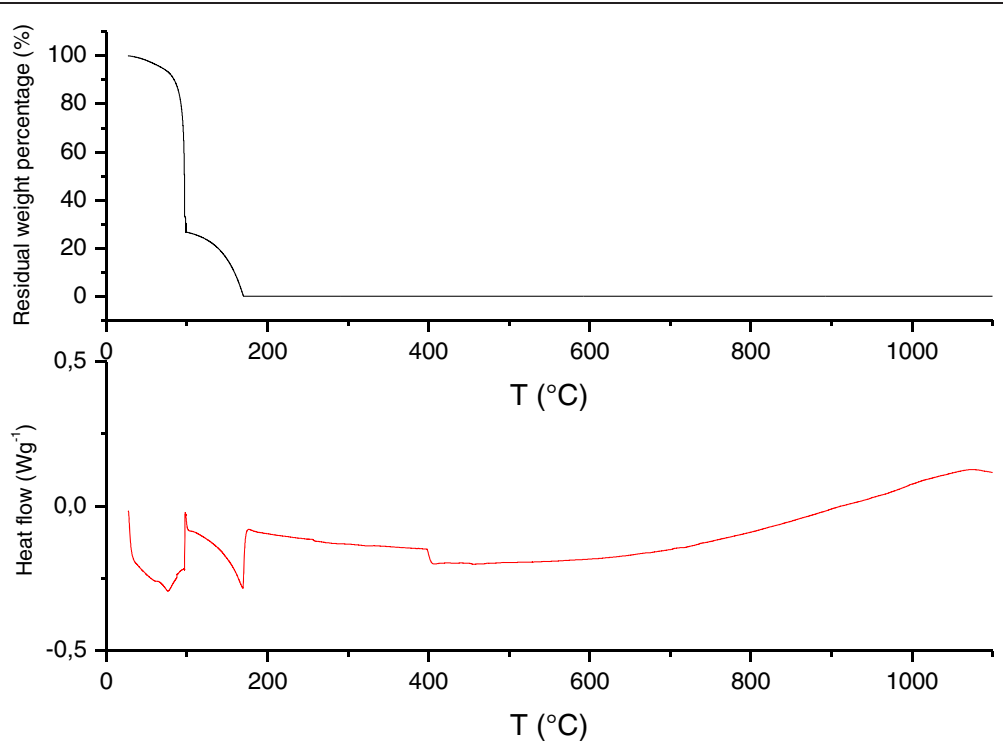

Figure 8 TGA/DSC of decalin.

\section{Conclusions}

The results of the thermogravimetric investigation of the new solid curing agents for polyurethane foams termed GreenCaps complement previous recent work $^{1,2}$ showing the large potential of organosilica microspheres encapsulating aqueous glycerol as solid curing agents. The TGA/DSC analyses presented in this account confirm the efficacy of glycerol sol-gel microencapsulation in $\mathrm{SiO}_{2}$ as well as in methylsilica particles until $10 \%$ degree of methylation. Typically, along with a $35 \mathrm{wt} \%$ of glycerol, the microspheres contain $c a$. $5 \mathrm{wt} \%$ encapsulated water.
The residual inorganic content of silica of the GreenCaps is around $40 \mathrm{wt} \%$, namely almost twice higher compared to core/shell $\mathrm{SiO}_{2}$ microcapsules encapsulating glycerol described by Galgali and co-workers. ${ }^{16}$ From the practical viewpoint of application of the GreenCaps in pressurized cans where stability for at least 12 months at room temperature is compulsory, the higher mechanical strenght of full microspheres compared to core-shell microcapsules is crucially relevant. All these findings are of relevant value in the development of silica-based porous microparticles encapsulating polyols and related species as solid curing agents for thermoset polymer resins.

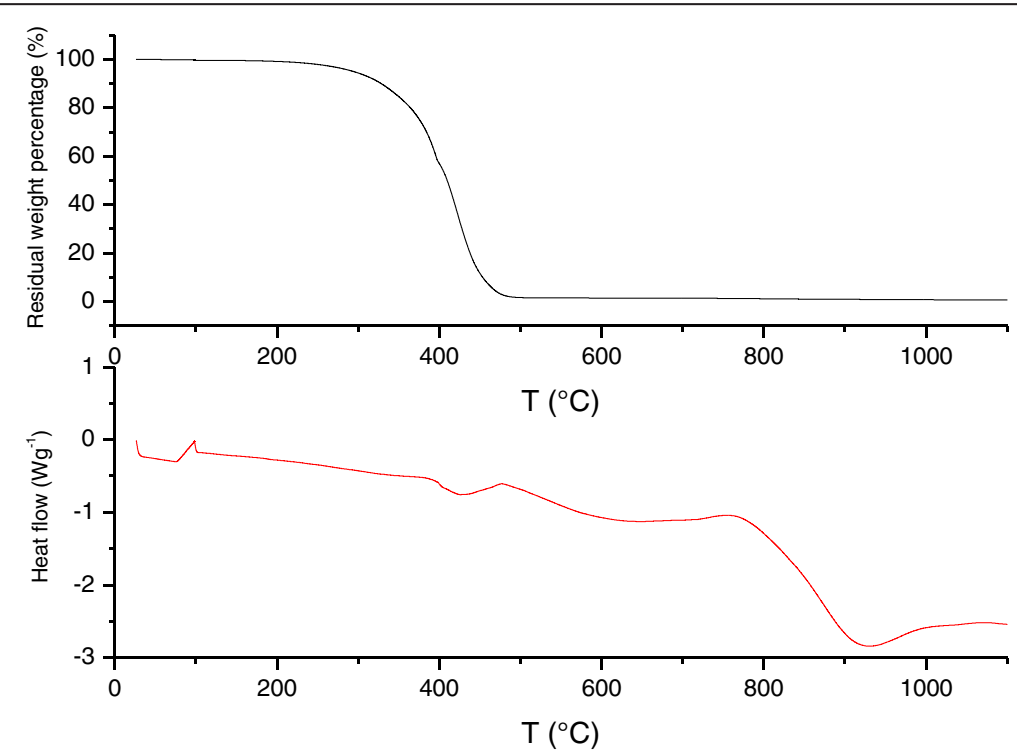

Figure 9 TGA/DSC of Span 80. 


\section{Competing interests}

The authors declare that they have no competing interests.

\section{Authors' contributions}

RC prepared the materials and drafted the manuscript. RLM participated in the materials preparation. GP participated in the design of the study and performed the TG analysis. RC and MP conceived of the study, and participated in its design and coordination and helped to draft the manuscript. All authors read and approved the final manuscript.

\section{Acknowledgements}

This article is dedicated to Professor Horst Böttcher (GMBU, Dresden) for his remarkable contribution to the progress of sol-gel microencapsulation. We thank Mr Aster De Schrijver (Greenseal Chemicals, Ghent), Dr Ana C. Marques (Greenseal Chemicals Research Center, Lisboa) and Professor João C.

Bordado (Instituto Superior Técnico, Lisboa) for purposeful co-operation.

Received: 18 September 2014 Accepted: 11 December 2014

Published online: 30 December 2014

\section{References}

1. Ciriminna R, Marques AC, Bordado JC, de Schrijver A, Pagliaro M, GreenCaps: Towards solid curing agents for sustainable polyurethane foams. Sustain Chem Processes 2014, 2:24.

2. Ciriminna R, Alterman A, Loddo V, De Schrijver A, Pagliaro M: Enhanced one-component spray polyurethane foams via sol-gel microspheres doped with aqueous glycerol. ACS Sustainable Chem Eng 2014, 2:506-511.

3. Tadros TF: Applied surfactants: principles and applications. Weinheim: Wiley-VCH; 2005:10. Chapter 1.

4. Ashida K: Polyurethane and related foams: chemistry and technology. Boca Raton: CRC Press; 2007:91. Chapter 4.

5. IAL Consultants: Global Overview of the Spray Polyurethane Foam (SPF) \& One Component Foam (OCF). London: October 2014.

6. US Environmental Protection Agency, Methylene Diphenyl Diisocyanate (MDI) and Related Compounds Action Plan Summary, November 2013. Available at the URL: http://www.epa.gov/oppt/existingchemicals/pubs/actionplans/mdi.html.

7. Meckel-Jonas C: (Henkel), Polyurethane Laminating Adhesives and Food Safety: A Smart Alliance. New Delhi: TAPPI Flexible Packaging Symposium India; 2009. 13-14 November 2009.

8. Hu C, Wan Y, Li: Production and characterization of biopolyols and polyurethane foams from crude glycerol based liquefaction of soybean straw. Bioresour Technol 2012, 103:227-233.

9. Luo $X$, Hu S, Zhang X, Li Y: Thermochemical conversion of crude glycerol to biopolyols for the production of polyurethane foams. Bioresour Technol 2013, 139:323-329.

10. Ciriminna R, Della Pina C, Rossi M, Pagliaro M: Understanding the Glycerol Market. Eur J Lipid Sci Technol 2014, 116:1432-1439.

11. Gómez EF, Luo X, Li C, Michel FC Jr, Li Y: Biodegradability of crude glycerol-based polyurethane foams during composting, anaerobic digestion and soil incubation. Polym Degrad Stabil 2014, 102:195-203.

12. Handbook of industrial surfactants. Ash M, Ash I (Eds), 4th edition, Synapse Information Resources: 2005.

13. Barbé CJA, Finnie KS, Kong L: Particles having hydrophobic material therein WO2006133518A1.

14. See at the URL: http://www.merck-performance-materials.com/en/ cosmetics/eusolex/eusolex_uv_pearls/eusolex_uv_pearls.html.

15. Rottman C, Grader GS, De Hazan Y, Avnir D: Sol-gel entrapment of ET (30) in ormosils. Interfacial polarity-fractality correlation. Langmuir 1996, 12:5505-5508.

16. Lobnik A, Wolfbeis OS: Polarity studies on ormosils using a solvatochromic fluorescent probe. Analyst 1998, 123:2247-2250.

17. Galgali G, Schlangen E, van der Zwaag S: Synthesis and characterization of silica microcapsules using a sustainable solvent system template. Mater Res Bull 2011, 46:2445-2449.

\section{Publish with ChemistryCentral and every scientist can read your work free of charge \\ "Open access provides opportunities to our colleagues in other parts of the globe, by allowing anyone to view the content free of charge." \\ W. Jeffery Hurst, The Hershey Company. \\ - available free of charge to the entire scientific community \\ - peer reviewed and published immediately upon acceptance \\ - cited in PubMed and archived on PubMed Central \\ - yours - you keep the copyright \\ Submit your manuscript here: \\ http://www.chemistrycentral.com/manuscript/<smiles>c1ccccc1</smiles> \\ Chemistry Central}

